\title{
SUBWAVELENGTH GRATINGS FOR GENERATING AZIMUTHALLY POLARIZED BEAMS
}

\author{
S.S. Stafeev ${ }^{1,2}$, M.V. Kotlyar ${ }^{2}$, L. O'Faolain ${ }^{3}$, A.G. Nalimov ${ }^{1,2}$, \\ V.V. Kotlyar ${ }^{1,2}$ \\ ${ }^{1}$ Image Processing Systems Institute - Branch of the Federal Scientific Research Centre "Crys- \\ tallography and Photonics" of Russian Academy of Sciences, Samara, Russia \\ ${ }^{2}$ Samara National Research University, Samara, Russia \\ ${ }^{3}$ School of Physics \& Astronomy, University of St. Andrews, Scotland, UK
}

\begin{abstract}
We have experimentally investigated two binary subwavelength grating-micropolarizers that transform linearly polarized light to the azimuthally polarized beam. The first micropolarizer operates in reflective mode (for wavelength $532 \mathrm{~nm}$ ) and was manufactured in a gold film. The second micropolarizer operates in transmitting mode (for wavelength $633 \mathrm{~nm}$ ) and was manufactured in silicon.
\end{abstract}

Keywords: subwavelength grating, cylindrical vector beam, azimuthal polarization.

Citation: Stafeev SS, Kotlyar MV, O'Faolain L, Nalimov AG, Kotlyar VV. Subwavelength gratings for generating azimuthally polarized beams. CEUR Workshop Proceedings, 2016; 1638: 125-131. DOI: 10.18287/1613-0073-20161638-125-131

\section{Introduction}

A significant amount of scientific papers describes investigation of beams with spatially non-homogeneous states of polarization [1]. In recent years subwavelength grating are used to obtain such beams. A subwavelength grating with properly chosen height of relief could operate as a half- (or quarter-) wave plate; meanwhile, the groove direction defines the spatial orientation of the plate [2].

As the first publications to report on the fabrication of the gratings, we may denote refs. [3,4], where a circularly polarized $10.6 \mu \mathrm{m}$ light beam was converted into an azimuthally polarized beam. The generation of a radially polarized light beam for a subwavelength grating operating at a $1064 \mathrm{~nm}$ wavelength was discussed in ref. [5]. A technique for fabricating a polarization converter in silicon for wavelengths ranging from 1030 to $1060 \mathrm{~nm}$ was proposed in ref. [6]. Transmitting micropolarizers for the visible range were also investigated. In ref. [7] aluminum circular subwavelength grating was used to convert circularly polarized light with wavelength $633 \mathrm{~nm}$ into radially polarized beam. However the quarter-wave plate was manufactured in [7] and 
the obtained beam was not strictly radially polarized - at diametrically opposite points of the beam the electric field has the same phase, while in the real radially polarized beam electric field at these points have opposite phase. Earlier, we discussed a 4-zone grating polarizer intended to form a radially polarized beam [8], which was used to form a subwavelength focal spot of area $0.35 \lambda \times 0.38 \lambda$ by means of a Fresnel zone plate [9].

In our investigation we have experimentally investigated two binary subwavelength grating-micropolarizers that transform linearly polarized light to the azimuthally polarized beam. The first micropolarizer operates in reflective mode and was manufactured in a gold film. The second micropolarizer operates in transmitting mode and was manufactured in silicon.

\section{Fabrication}

The proposed reflective micropolarizer consists of four sectors (Fig. 1(a)): in the two sectors on the right, the microrelief features have a $0.46 \mu \mathrm{m}$ period and make angles $70^{\circ}$ and $-70^{\circ}$ with the y-axis (vertical), whereas in the sectors on the left, the features have a $0.4 \mu \mathrm{m}$ period and make with the y-axis angles $40^{\circ}$ and $-40^{\circ}$. The micropolarizer has a size of $100 \mu \mathrm{m} \times 100 \mu \mathrm{m}$ and the microrelief height of $110 \mathrm{~nm}$. The micropolarizer was fabricated by electron beam lithography in a golden layer of thickness $160-180 \mathrm{~nm}$. In the first step of fabrication a golden layer was coated onto a glass substrate, followed by applying a resist layer, on which a four-sector grating polarizer pattern was generated by an electron beam lithography (at $30 \mathrm{kV}$ ). Then, the sample was developed by etching with xylene, which dissolved the fragments exposed to the electron beam. Then, using reactive ion etching, the grating-polarizer pattern was transferred into the golden layer, which was etched in places with no resist. Using argon plasma, gold particles were spattered from areas unprotected with the resist. At the final stage, the resist residue was eliminated using oxygen plasma. The reactive ion etching time was optimized so as to achieve an etch depth of about $110 \mathrm{~nm}$.

The transmitting micropolarizer (Fig. 1(b)) also consists of four sectors with angles $60^{\circ}, 60^{\circ},-60^{\circ}, 60^{\circ}$. The micropolarizer not only transforms linearly polarized light into azimuthally polarized beam but also adds phase shift $\pi$ at diametrically opposite points of the beam. The micropolarizer was fabricated by electron beam lithography. In the first step of manufacturing a layer of amorphous silicon $(130 \mathrm{~nm})(\mathrm{a}-\mathrm{Si})$ was coated with a resist (PMMA). The thickness of the resist was chosen optimally (320 $\mathrm{nm}$ ). The substrate was coated by $15 \mathrm{~nm}$ of gold to avoid the formation of the charge on the sample surface. A four-sector grating polarizer pattern was generated by an electron beam lithography (at $30 \mathrm{kV}$ ). Then the substrate was developed using solution of water and isopropanol (ratio of 3:7). During this process, the gold layer was completely removed from the surface of the PMMA. Using reactive ion etching the pattern of grating-polarizer was transferred from the resist to amorphous silicon. The manufactured micropolarizer have period $230 \mathrm{~nm}$, step width $138 \mathrm{~nm}$, and groove width $92 \mathrm{~nm}$. The size of the manufactured micropolarizer (Fig. 1b) is $100 \times 100 \mu \mathrm{m}$. 

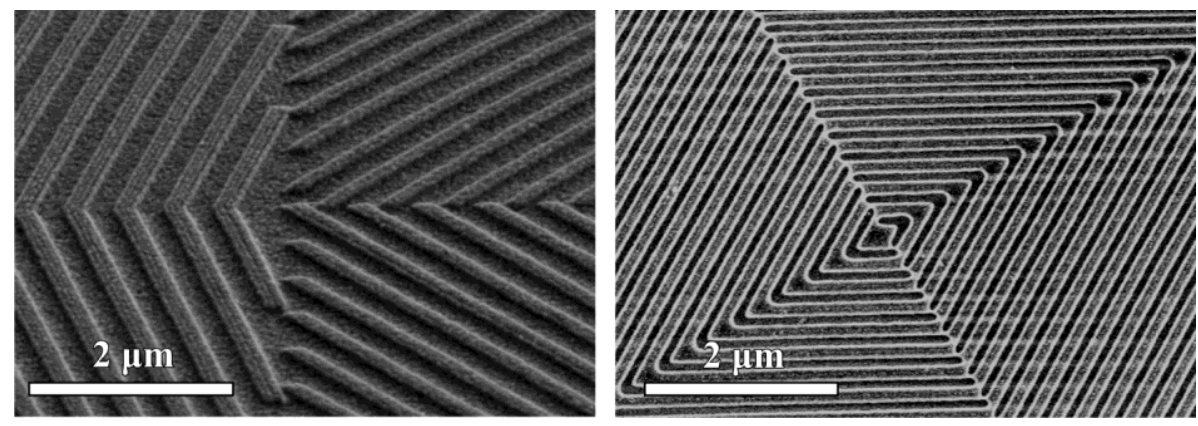

Fig. 1. A scanning electron microscope image of the central fragment of the fabricated (a) reflective and (b) transmitting micropolarizers

\section{Experiment}

Reflective polarizer

The performance of the fabricated reflective micropolarizer (fig. 1a) was experimentally tested using a linearly polarized beam of $1 \mathrm{~mm}$ width from a $532 \mathrm{~nm}$ laser. The beam was focused with a $10 \times$ lens $\mathrm{O}_{1}$ onto the substrate containing the grating micropolarizer. Experimental setup is shown in fig. 2. The size of the spot focused on the micropolarizer was controlled by varying the distance from the lens $\mathrm{O}_{1}$ to the micropolarizer surface. Although in this case, the micropolarizer was not found in the beam waist and the incident wave was spherical, the experimental results we discuss below corroborate that the micropolarizer still operated in a proper way.

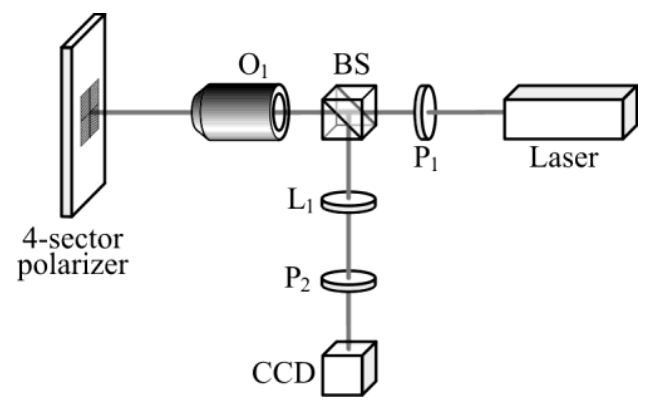

Fig. 2. Experimental setup: $P_{1}, P_{2}$ are linear polarizers, $B S$ is a beam-splitter cube, $\mathrm{O}_{1}$ is a $10 \times$ lens $(\mathrm{NA}=0.25), \mathrm{L}_{1}$ is a lens $(f \approx 1.5 \mathrm{~cm})$, and CCD is a camera

Fig. 3 shows intensity of the laser beam reflected from polarizer. A linear polarizer $\mathrm{P}_{2}$ is located before the camera; an angle between the axis of polarizer and incident light polarization equals to $0^{\circ}$ (a), $90^{\circ}$ (b), $-45^{\circ}$ (c) and $45^{\circ}$ (d). Fig 3 shows that light reflected from 4 -sector micropolarizer is azimuthally polarized. 


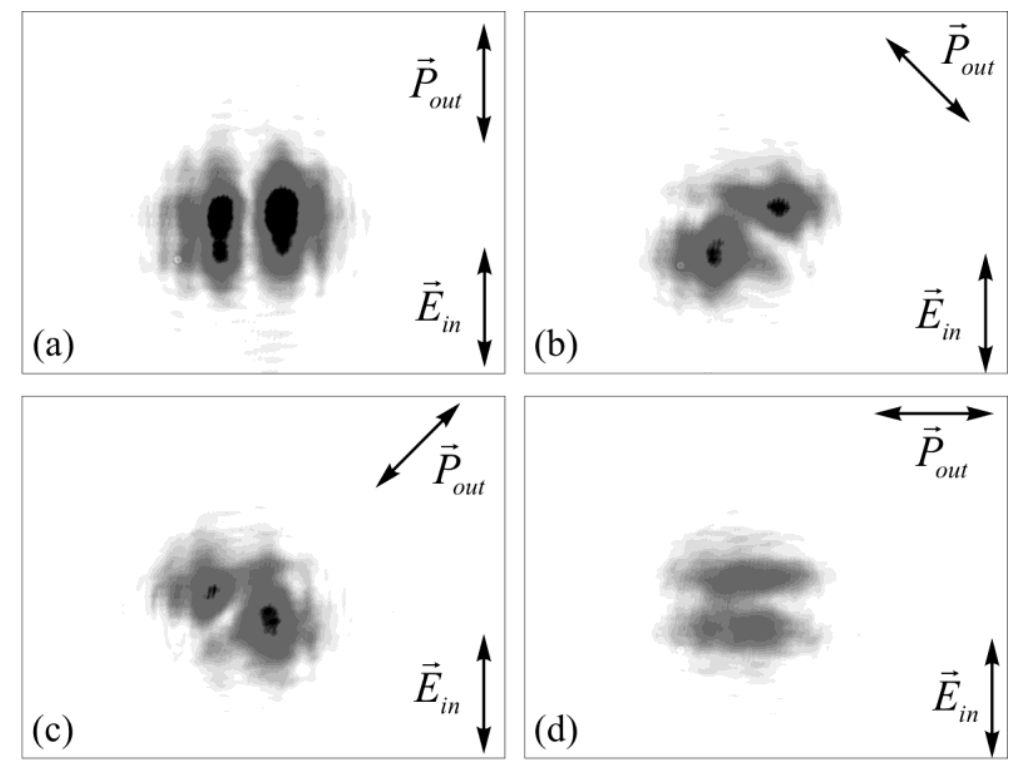

Fig. 3. Image of the laser beam reflected from polarizer (Fig. 1a) in far field. A linear polarizer is located before the camera; an angle between the axis of polarizer and incident light polarization equals to $0^{\circ}$ (a), $90^{\circ}$ (b), $-45^{\circ}$ (c) and $45^{\circ}$ (d). Image size is $6.2 \mathrm{~mm} \times 4.2 \mathrm{~mm}$

\section{Transmitting polarizer}

The performance of the transmitting micropolarizer (Fig. 1b) was experimentally tested using a linearly polarized beam from a $633 \mathrm{~nm}$ laser. The beam was focused with a $40 \times$ lens $\mathrm{O}_{1}$ onto the substrate containing the grating micropolarizer (Fig. 4). The size of the spot focused on the micropolarizer was controlled by varying the distance from the lens $\mathrm{O}_{1}$ to the micropolarizer surface. The substrate was mounted on a movable stage, and the position of the light spot on the micropolarizer was controlled by shifts of the stage. The CCD camera image was obtained using a $20 \times$ lens $\mathrm{O}_{2}$. As previously, linear polarizer $\mathrm{P}_{2}$ is located before the camera to check a polarization state of the reflected beam.

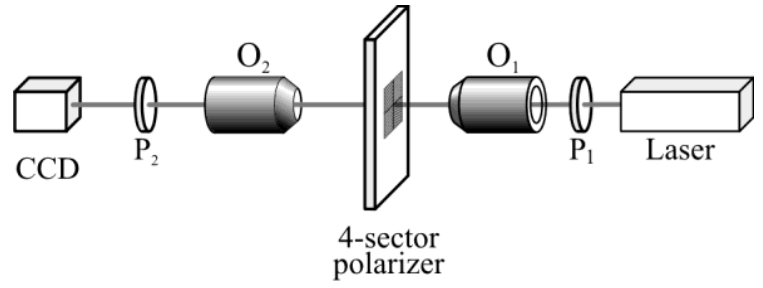

Fig. 4. Experimental setup: Laser is a He-Ne laser, $\mathrm{P}_{1}, \mathrm{P}_{2}$ are linear polarizers, $\mathrm{O}_{1}$ is a $40 \times$ lens, and $\mathrm{O}_{2}$ is a $10 \times$ lens

Fig. 5 shows image of the transmitting micropolarizer in Fig. 1b in the laser light. An angle between the axis of output polarizer $\mathrm{P}_{2}$ and incident light polarization equals to 
$0^{\circ}$ (a), $90^{\circ}(\mathrm{b}),-45^{\circ}(\mathrm{c})$ and $45^{\circ}$ (d). Based on fig. 5 , we can infer that the four-sector micropolarizer in fig. 1(b) converts an incident linearly polarized beam into an azimuthally polarized light beam.
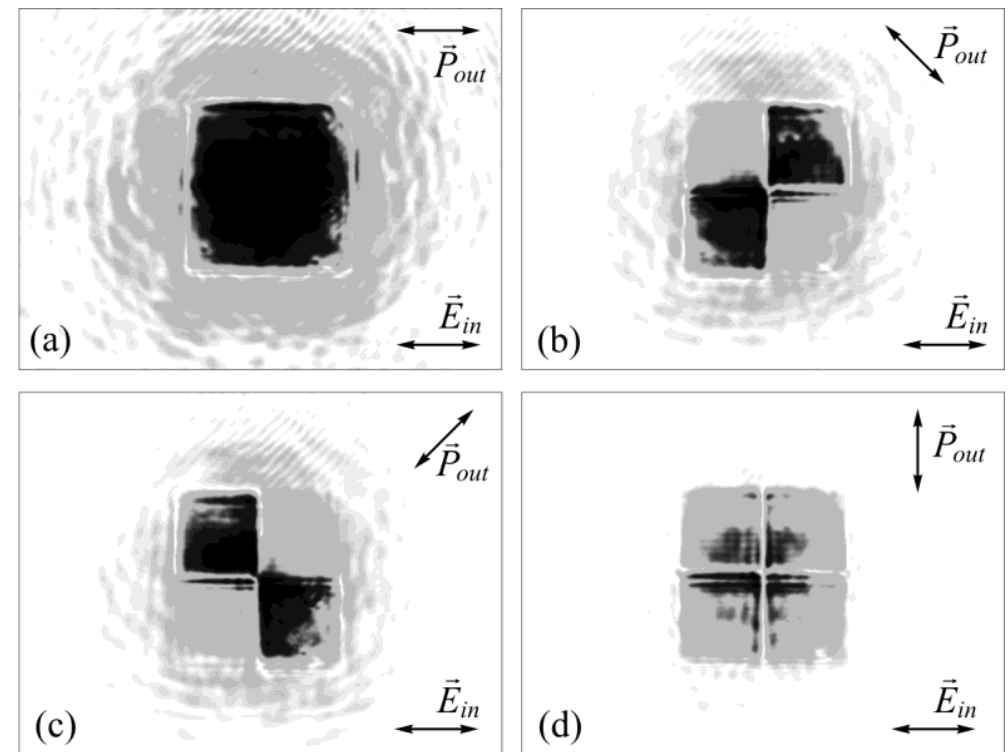

Fig. 5. Image of the micropolarizer in Fig. $1 b$ in the laser light. In front of the camera was an output polarizer/analyzer $\mathrm{P}_{2}$, making an angle of (a) $0^{\circ}$, (b) $90^{\circ}$, (c) $-45^{\circ}$, and (d) $45^{\circ}$ with the incident beam

A lens with a focal length of $24 \mathrm{~mm}$ was placed in front of the polarizer $\mathrm{P}_{2}$ (in Fig. 4) to investigate the intensity distributions in the far field. Fig. 6 shows intensity of the laser beam in far field. The micropolarizer forms a peak of intensity on the optical axis. A central lobe has a circular shape, and a side lobe has a form of non-uniform ring due to the square shape of the micropolarizer. Based on fig. 6 we can infer that the micropolarizer not only convert linearly polarized light into azimuthally polarized beam but also add the phase shift $\pi$ to diametrically opposite points of the beam [1014].

\section{Conclusions}

In this work we have investigated two subwavelength gratings that transform linearly polarized light into azimuthally polarized beam. The first micropolarizer operates in reflective mode and was manufactured in a gold film. The second micropolarizer operates in transmitting mode and was manufactured in silica. It was shown experimentally the following: 
1) Manufactured 4-sector reflective micropolarizer illuminated by linearly polarized light with wavelength of $532 \mathrm{~nm}$ forms azimuthally polarized beam in near and far field

2) Manufactured 4-sector transmitting micropolarizer illuminated by linearly polarized light with wavelength of $633 \mathrm{~nm}$ forms in near field azimuthally polarized beam with phase shift $\pi$ at diametrically opposite points of the beam. In far field of diffraction the micropolarizer forms a central circular focal spot surrounded by a light ring.
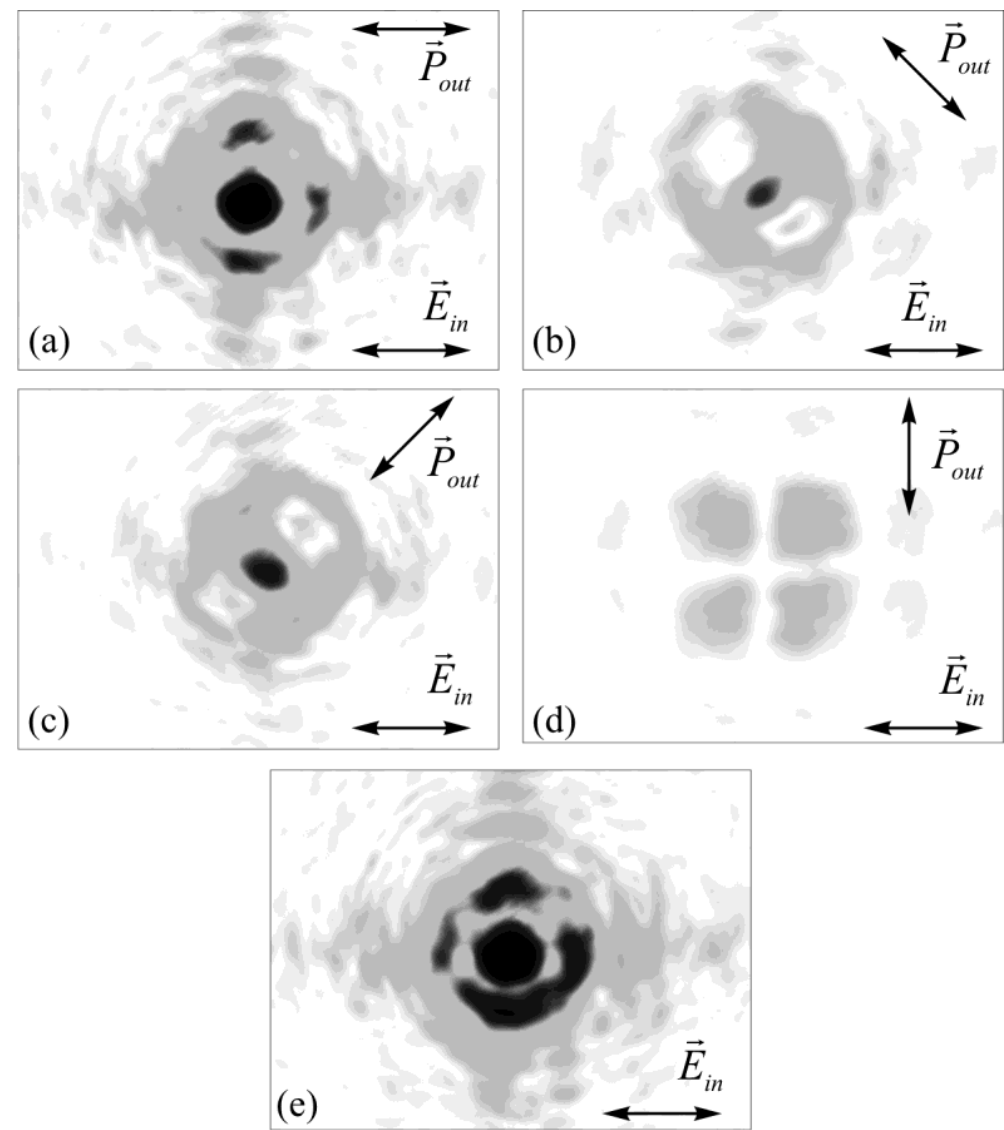

Fig. 6. Image of the laser beam transmitted through the polarizer (Fig. 1b) in far field. A linear polarizer is located before the camera; an angle between the axis of polarizer and incident light polarization equals to $0^{\circ}(\mathrm{a}), 90^{\circ}(\mathrm{b}),-45^{\circ}(\mathrm{c})$ and $45^{\circ}(\mathrm{d})$. Image size is $2.7 \mathrm{~mm} \times 2.0 \mathrm{~mm}$

\section{Acknowledgments}

This work was partially funded by the Ministry of Education and Science of the Russian Federation and Russian Federation Presidential grants for support of Young Candidates of Science (MK-9019.2016.2), and Russian Foundation for Basic Research 
grants \#\# 14-29-07133, 14-07-97039, 15-07-01174, 15-37-20723, 15-47-02492, 16 07-00990.

\section{References}

1. Zhan Q. Cylindrical vector beams: from mathematical concepts to applications. Adv. Opt. Photon, 2009; 1: 1-57.

2. Kotlyar VV, Zalyalov OK. Design of diffractive optical elements modulating polarization. Optik, 1996; 103(3): 125-130.

3. Bozom Z, Kleiner V, Hasman E. Pancharatnam-Berry phase in space-variant polarizationstate manipulations with subwavelengtn gratings. Optics Letters, 2001; 26(18): 1424-1426.

4. Bozom Z, Biener G, Kleiner V, Hasman E. Radially and azimutally polarized beams generated by space-variant dielectric subwavelength gratings. Optics Letters, 2002; 27(5): 285-287.

5. Lerman GM, Levy U. Generation of a radially polarized light beam using space-variant subwavelength gratings at $1064 \mathrm{~nm}$. Optics Letters, 2008; 33(23): 2782-2784.

6. Kämpfe T, Sixt P, Renaud D, Lagrange A, Perrin F, Parriaux O. Segmented subwavelength silicon gratings manufactured by high productivity microelectronic technologies for linear to radial/azimuthal polarization conversion. Optical Engineering, 2014; 53(10): 107105.

7. Ghadyani Z, Vartiainen I, Harder I, Iff W, Berger A, Lindlein N, Kuittinen M. Concentric ring metal grating for generating radially polarized light. Applied Optics, 2011; 50(16): 2451-2457.

8. Nalimov AG, O'Faolain L, Stafeev SS, Shanina MI, Kotlyar VV. Reflected four-zones subwavelength mictooptics element for polarization conversion from linear to radial. Computer Optics, 2014; 38(2): 229-236.

9. Stafeev SS, O'Faolain L, Kotlyar VV, Nalimov AG Tight focus of light using micropolarizer and microlens. Applied Optics, 2015; 54(14): 4388-4394.

10. Helseth LE. Optical vortices in focal regions. Optics Communications, 2004; 229: 85-91.

11. Zhang Z, Pu J, Wang X. Tight focusing of radially and azimuthally polarized vortex beams through a uniaxial birefringent crystal. Applied Optics, 2008; 47: 1963-1967

12. Hao X, Kuang C, Wang T, Liu X. Phase encoding for sharper focus of the azimuthally polarized beam. Optics Letters, 2010; 35: 3928-3930.

13. Wang S, Li X, Zhou J, Gu M. Ultralong pure longitudinal magnetization needle induced by annular vortex binary optics. Optics Letters, 2014; 39: 5022-5025.

14. Li X, Venugopalan P, Ren H, Hong M, Gu M. Super-resolved pure-transverse focal fields with an enhanced energy density through focus of an azimuthally polarized first-order vortex beam. Optics Letters, 2014; 39: 5961-5964. 